\title{
Successful treatment of a 19-year-old patient with locally advanced clear cell adenocarcinoma of the uterine cervix using recombinant human adenovirus type 5 (Oncorine) combined with chemoradiotherapy: a case report
}

\author{
Jing Zhang, Zi Liu, Qi-Ying Zhang, Tao Wang, Juan Wang, Fan Shi, Jin Su, Tuo-Tuo Gong \\ Department of Radiation Oncology, The First Affiliated Hospital of Xi'an Jiao Tong University, Xi'an, China \\ Correspondence to: Zi Liu. Department of Radiation Oncology, The First Affiliated Hospital of Xi'an Jiao Tong University, No. 277, West Yanta Road, \\ Xi’an 710061, China. Email: liuzmail@126.com.
}

\begin{abstract}
Clear cell adenocarcinoma (CCAC) of the uterine cervix is a rare histological subtype of cervical cancer. The optimal treatment paradigm for this tumor has not yet been established. In recent years, oncolytic viruses have become a promising strategy for cancer treatment. However, the role of oncolytic viruses in treating CCAC of the uterine cervix is unclear. We report a case study of a 19-year-old woman with locally advanced CCAC of the uterine cervix. The patient was first treated with external beam radiation therapy (50 Gy/25 f) and chemotherapy (cisplatin, $40 \mathrm{mg} / \mathrm{m}^{2} /$ week, 5 weeks) with only slight reduction of the cervical tumor $(45 \mathrm{~mm} \times 34 \mathrm{~mm} \times 51 \mathrm{~mm})$. After receiving one cycle of intratumor injection therapy of Oncorine $\left(5.0 \times 10^{11}\right.$ virus particles daily, from day 1 to day 5$)$, her cervical tumor was dramatically reduced $(29 \mathrm{~mm} \times 26 \mathrm{~mm} \times 24 \mathrm{~mm})$. Subsequently, the patient received two cycles of intratumor injection therapy of Oncorine combined with brachytherapy ( 7 fractions) and chemotherapy (cisplatin and paclitaxel). No serious adverse events occurred during treatment. The results at the 7 -month follow-up showed that the patient achieved complete response. Our case was a successful exploration of Oncorine in the treatment of locally advanced CCAC of the uterine cervix, which supports the use of oncolytic viruses as a promising treatment option for young women with this tumor.
\end{abstract}

Keywords: Case report; chemoradiotherapy; clear cell adenocarcinoma (CCAC); Oncorine; uterine cervix

Submitted Aug 31, 2021. Accepted for publication Dec 01, 2021.

doi: $10.21037 / \mathrm{atm}-21-5963$

View this article at: https://dx.doi.org/10.21037/atm-21-5963

\section{Introduction}

Clear cell adenocarcinoma (CCAC) of the uterine cervix is a rare and highly invasive histological subtype of cervical cancer (1). Its pathogenesis is usually associated with in utero exposure to diethylstilbestrol (2). Although most patients are diagnosed with CCAC at stage I or stage II, the 5 -year survival rate was only $55.7 \%$ (3). The optimal treatment paradigm for this tumor has not yet been established. Oncolytic viruses are designed to selectively replicate in cancer cells and kill tumor cells by inducing viral oncolysis without damaging normal tissue (4). Although limited to some extent by the way of administration, oncolytic virus therapy has become a promising strategy for cancer treatment recently (5). The anti-cancer activity of oncolytic virus results from two distinct mechanisms: selective replication in tumor cells, resulting in a direct lytic effect on tumor cells; and induction of systemic anti-tumor immunity (5). Oncorine (H101) is the first commercialized oncolytic virus in the world, and was approved by the National Medical Products Administration of China in 2005 for the treatment of nasopharyngeal carcinoma (6). It has exhibited anticancer properties in several types of tumors, including hepatocellular carcinoma, non-small cell lung cancer, and squamous cell cancer of the head and 
neck or esophagus (7-9). However, the role of Oncorine in the treatment of CCAC of the uterine cervix is unclear. In this article, we report a case study of a patient with locally advanced CCAC of the uterine cervix who was successfully treated with Oncorine combined with chemoradiotherapy. We present the following article in accordance with the CARE reporting checklist (available at https://dx.doi. org/10.21037/atm-21-5963).

\section{Case presentation}

In May 2020, a 19-year-old unmarried woman presented with a history of abnormal vaginal bleeding for 11 months and was referred to the Second Affiliated Hospital of Air Force Medical University. She reached menarche at the age of 13 years and had regular menstrual cycles before the onset of the symptom. The patient had no history of exposure to diethylstilbestrol, sexual intercourse, or smoking.

Magnetic resonance imaging (MRI) revealed a $50 \mathrm{~mm} \times 38 \mathrm{~mm} \times 61 \mathrm{~mm}$ tumor mass in the uterine cervix (Figure 1A). A gynecological examination suggested advanced CCAC of the uterine cervix (stage IIIB). The patient received external beam radiation therapy (50 Gy/25 f) combined with chemotherapy (cisplatin, $40 \mathrm{mg} / \mathrm{m}^{2} /$ week) for 5 weeks. However, MRI on June 17 (Figure $1 B$ ) demonstrated that the tumor mass was only slightly reduced $(45 \mathrm{~mm} \times 34 \mathrm{~mm} \times 51 \mathrm{~mm})$.

The same day, the patient was referred to The First Affiliated Hospital of Xi'an Jiaotong University. A gynecological examination showed that the residual cervical tumor was huge and the cervix was not exposed (Figure 1C); thus, standard brachytherapy would have been difficult to administer normally. After informed consent was obtained from the patient and her guardians, the patient received an experimental treatment of Oncorine (intratumor injection; $1.0 \times 10^{12}$ virus particles daily; Sunway Biotech, China) for 5 days (1 cycle). MRI (Figure 1D) on June 21 showed a dramatic reduction in the size of the expansive lesion (29 $\mathrm{mm} \times 26 \mathrm{~mm} \times 24 \mathrm{~mm}$ ). Additionally, a gynecological examination (Figure 1E) showed that part of the tumor had naturally shed and the cervix was exposed. Since June 22, the patient received 7 fractions ( $2 \mathrm{f} /$ week) of computed tomography-guided brachytherapy. The total equivalent dose in 2 Gy/f (EQD2) at point A (external beam plus brachytherapy) was 92.75 Gy. A gynecological examination on July 13 revealed a further reduction in the cervical tumor mass (Figure 1F). Thereafter, the patient received 2 cycles of intratumor injection therapy of Oncorine $\left(5.0 \times 10^{11}\right.$ virus particles daily, from day 1 to day 5) and 6 cycles of cisplatin $\left(40 \mathrm{mg} / \mathrm{m}^{2}\right.$, day 1 , every 3 weeks) and paclitaxel $\left(150 \mathrm{mg} / \mathrm{m}^{2}\right.$, day 1 , every 3 weeks) combination chemotherapy. After 2 cycles of the combination chemotherapy and 2 cycles of the intratumor injection therapy of Oncorine, the cervical tumor mass was further reduced (Figure 1G,1H). No serious adverse events occurred during treatment.

One month after the end of chemotherapy, the patient was reassessed by MRI, which showed no definite lesion in the cervix (Figure 2A). At the same time, the gynecological examination revealed the disappearance of the cervical tumor and the elastic recovery of the parauterine tissue (Figure 2B). Subsequently, the results at the 7-month followup appointment showed that the patient had achieved a complete response (Figure 2C,2D).

All procedures performed in this study involving human participants were in accordance with the ethical standards of the institutional and/or national research committee(s) and with the Helsinki Declaration (as revised in 2013). Written informed consent was obtained from the patient for publication of this case report and accompanying images. A copy of the written consent is available for review by the editorial office of this journal.

\section{Discussion}

In this article, we reported a case study of a 19-year-old woman with locally advanced CCAC of the uterine cervix (stage IIIB). After trying external beam radiation therapy plus chemotherapy, the cervical tumor mass of the patient only reduced slightly. Subsequently, the patient received combination therapy of Oncorine and chemoradiotherapy, and finally achieved a complete response. This appears to be the first case report on the efficacy of Oncorine in the treatment of advanced CCAC of the uterine cervix.

The standard treatment for locally advanced CCAC of the uterine cervix includes pelvic external beam radiation therapy, chemotherapy, and brachytherapy (10). In our case, the residual cervical tumor was still huge after external beam radiotherapy and cisplatin-based chemotherapy, which made it difficult to implement brachytherapy. Thus, it was necessary to explore therapeutic methods other than the standard treatment.

In recent years, a number of combination therapeutic strategies were investigated to enhance the treatment effects of oncolytic virus $(11,12)$. A multicenter, phase $\mathrm{Ib}$ trial showed that the combination of oncolytic virus and 

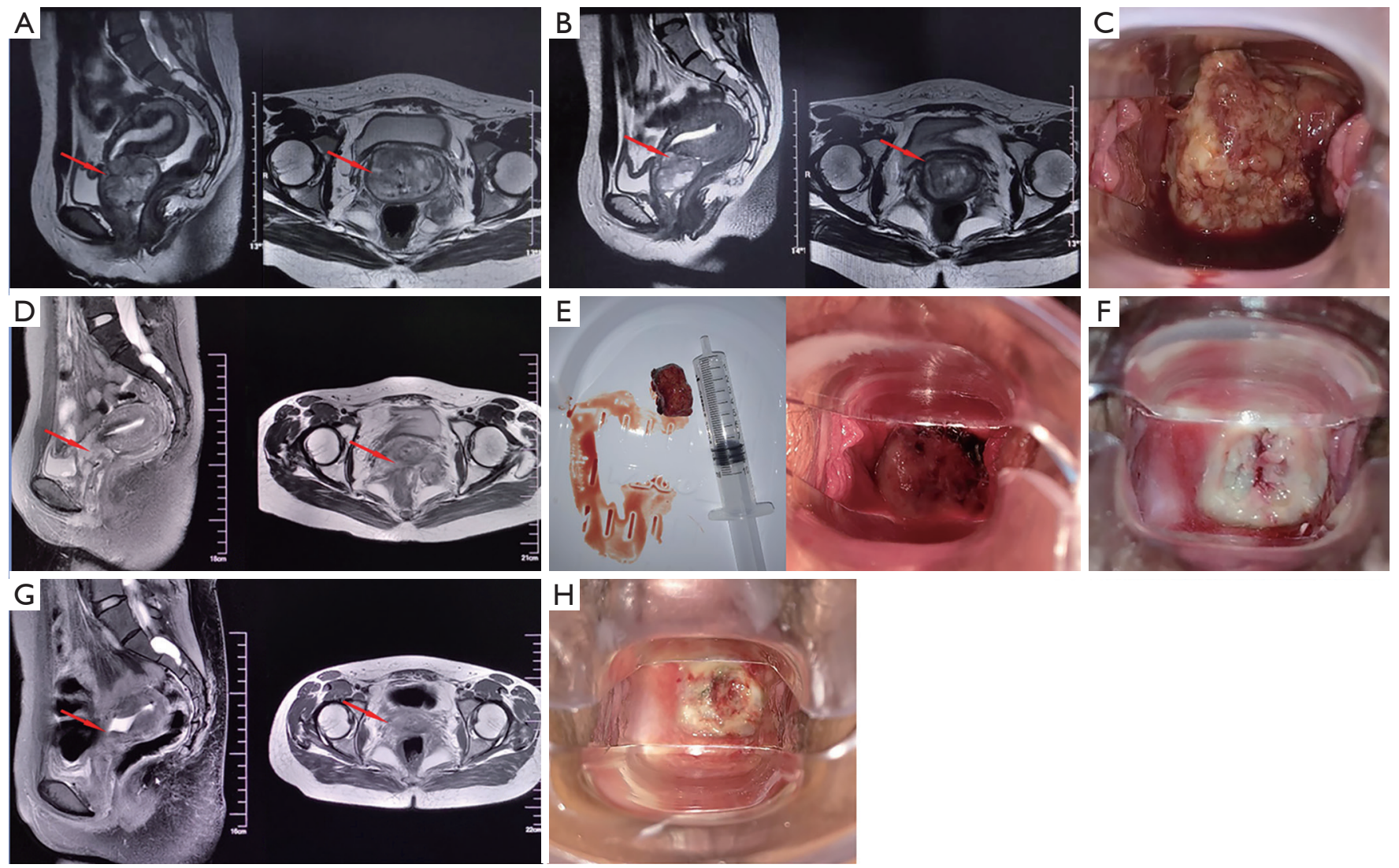

Figure $1 \mathrm{MRI}$ and gynecological examinations of the patient before and during treatment. (A) MRI before treatment revealed a $50 \mathrm{~mm} \times$ $38 \mathrm{~mm} \times 61 \mathrm{~mm}$ tumor mass (red arrow) in the uterine cervix. (B) MRI after external beam radiation therapy and chemotherapy demonstrated that the tumor mass (red arrow) was only slightly reduced (45 $\mathrm{mm} \times 34 \mathrm{~mm} \times 51 \mathrm{~mm}$ ). (C) A gynecological examination after external beam radiation therapy and chemotherapy showed that the residual cervical tumor was huge and the cervix was not exposed. (D) MRI after 5 days of intratumor injection therapy of Oncorine demonstrated a dramatic reduction in the size of the expansive lesion (29 mm $\times 26 \mathrm{~mm} \times 24 \mathrm{~mm}$, red arrow). (E) A gynecological examination after 5 days of intratumor injection therapy of Oncorine showed that part of the tumor had naturally shed and the cervix was exposed. (F) A gynecological examination after 7 fractions ( $2 \mathrm{f} /$ week) of brachytherapy demonstrated a further reduction in the cervical tumor mass. $(\mathrm{G}, \mathrm{H})$ After receiving 7 fractions (2 f/week) of brachytherapy, 2 cycles of chemotherapy, and 2 cycles of intratumor injection therapy of Oncorine, the cervical tumor mass was further reduced. The red arrow stands for the tumor mass.

ipilimumab appeared to have greater efficacy than either oncolytic virus or ipilimumab monotherapy in patients with advanced melanoma (11). Saha et al. fund that the combination of an oncolytic virus with two immune checkpoint inhibitors (ICIs) could eradicate glioma in two mouse models, and the therapeutic efficacy depended on CD4+, CD8+ T cells and macrophages (12). Oncorine, an oncolytic adenovirus with both the E1B and E3 gene deletions, can selectively target and kill tumor cells without damaging normal tissue (13). Several clinical studies have demonstrated the properties of Oncorine in enhancing the efficacy of other anti-tumor therapies (7,8). Compared with transarterial chemoembolization (TACE) therapy, patients who were diagnosed with unresectable hepatocellular carcinoma treated with combined TACE and Oncorine therapy had increased overall survival and decreased cancer-specific mortality (7). Besides, the role of Oncorine in reversing resistance to ICIs has been confirmed in a case with recurrent non-small cell lung cancer (8). After 1 cycle of intratumor injection therapy of Oncorine, the cervical tumor mass of the patient in our case reduced dramatically, which created the conditions necessary for the 

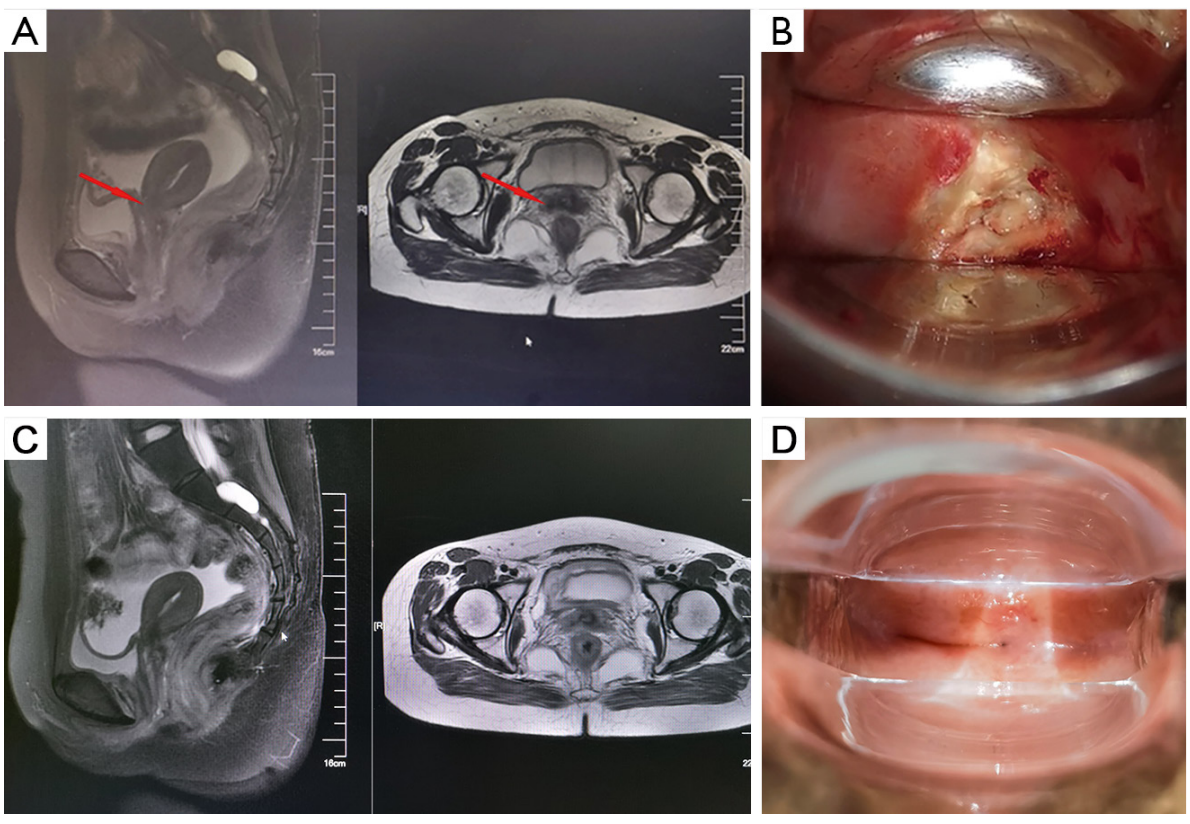

Figure $2 \mathrm{MRI}$ and gynecological examinations of the patient during the 7-month follow-up. (A) 1 month after the end of treatment, MRI showed no definite lesion in the cervix. The red arrow stands for the tumor mass. (B) 1 month after the end of treatment, a gynecological examination revealed the disappearance of the cervical tumor and the elastic recovery of the parauterine tissue. (C) MRI at the 7-month follow-up examination showed that the cervical outline had returned to normal. (D) A gynecological examination at the 7-month follow-up appointment showed that the local cervical scar had healed.

successful completion of brachytherapy. The patient then received 7 fractions ( $2 \mathrm{f} /$ week) of brachytherapy, 6 cycles of chemotherapy, and 2 cycles of intratumor injection therapy of Oncorine, and achieved a complete response without serious adverse events. Our study suggests that Oncorine combined with brachytherapy might be an effective treatment paradigm for intractable cervical tumors.

In this case, Oncorine was successfully administered in the treatment of locally advanced CCAC of the uterine cervix. These results support the use of oncolytic viruses as a promising treatment option for young women with this type of tumor. The efficacy and safety of oncolytic viruses combined with brachytherapy and chemotherapy for patients with intractable cervical tumors need to be validated in future studies.

\section{Acknowledgments}

We would like to thank Professor Qiu-Ju Shao and Doctor Jie-Jing Zhou (Tangdu Hospital of The Second Affiliated Hospital of Air Force Medical University) for their help in collecting the initial medical history data.
Funding: None.

\section{Footnote}

Reporting Checklist: The authors have completed the CARE reporting checklist. Available at https://dx.doi. org/10.21037/atm-21-5963

Conflicts of Interest: All authors have completed the ICMJE uniform disclosure form (available at https://dx.doi. org/10.21037/atm-21-5963). The authors have no conflicts of interest to declare.

Ethical Statement: The authors are accountable for all aspects of the work in ensuring that questions related to the accuracy or integrity of any part of the work are appropriately investigated and resolved. All procedures performed in this study involving human participants were in accordance with the ethical standards of the institutional and/or national research committee(s) and with the Helsinki Declaration (as revised in 2013). Written informed consent was obtained from the patient for publication of this case 
report and accompanying images. A copy of the written consent is available for review by the editorial office of this journal.

Open Access Statement: This is an Open Access article distributed in accordance with the Creative Commons Attribution-NonCommercial-NoDerivs 4.0 International License (CC BY-NC-ND 4.0), which permits the noncommercial replication and distribution of the article with the strict proviso that no changes or edits are made and the original work is properly cited (including links to both the formal publication through the relevant DOI and the license). See: https://creativecommons.org/licenses/by-nc-nd/4.0/.

\section{References}

1. Ferrandina G, Lucidi A, De Ninno M, et al. Successful treatment of a young patient with locally advanced clear cell adenocarcinoma of the uterine cervix undergoing chemoradiation followed by radical surgery. Gynecol Obstet Invest 2014;77:64-7.

2. Palaiologos K, Theofrastou SS, Gerovasileiou E, et al. A Rare Case of Clear Cell Cervical Carcinoma to a Woman, 50 Years After Diethylstilbestrol Exposure for Lactation Suppression. Cureus 2021;13:e17468.

3. Seki H, Takada T, Sodemoto T, et al. A young woman with clear cell adenocarcinoma of the uterine cervix. Int J Clin Oncol 2003;8:399-404.

4. Macedo N, Miller DM, Haq R, et al. Clinical landscape of oncolytic virus research in 2020. J Immunother Cancer 2020;8:e001486.

5. Wei D, Xu J, Liu XY, et al. Fighting Cancer with Viruses: Oncolytic Virus Therapy in China. Hum Gene Ther 2018;29:151-9.

6. Liang M. Oncorine, the World First Oncolytic Virus

Cite this article as: Zhang J, Liu Z, Zhang QY, Wang T, Wang J, Shi F, Su J, Gong TT. Successful treatment of a 19-year-old patient with locally advanced clear cell adenocarcinoma of the uterine cervix using recombinant human adenovirus type 5 (Oncorine) combined with chemoradiotherapy: a case report. Ann Transl Med 2021;9(23):1747. doi: 10.21037/atm-21-5963
Medicine and its Update in China. Curr Cancer Drug Targets 2018;18:171-6.

7. He C, Zhang Y, Lin X. Increased Overall Survival and Decreased Cancer-Specific Mortality in Patients with Hepatocellular Carcinoma Treated by Transarterial Chemoembolization and Human Adenovirus Type5 Combination Therapy: a Competing Risk Analysis. J Gastrointest Surg 2018;22:989-97.

8. Zhang QN, Li Y, Zhao Q, et al. Recombinant human adenovirus type 5 (Oncorine) reverses resistance to immune checkpoint inhibitor in a patient with recurrent non-small cell lung cancer: A case report. Thorac Cancer 2021;12:1617-9.

9. Xia ZJ, Chang JH, Zhang L, et al. [Phase III randomized clinical trial of intratumoral injection of $\mathrm{E} 1 \mathrm{~B}$ genedeleted adenovirus (H101) combined with cisplatin-based chemotherapy in treating squamous cell cancer of head and neck or esophagus]. Ai Zheng 2004;23:1666-70.

10. Robin TP, Amini A, Schefter TE, et al. Disparities in standard of care treatment and associated survival decrement in patients with locally advanced cervical cancer. Gynecol Oncol 2016;143:319-25.

11. Puzanov I, Milhem MM, Minor D, et al. Talimogene Laherparepvec in Combination With Ipilimumab in Previously Untreated, Unresectable Stage IIIB-IV Melanoma. J Clin Oncol 2016;34:2619-26.

12. Saha D, Martuza RL, Rabkin SD. Macrophage Polarization Contributes to Glioblastoma Eradication by Combination Immunovirotherapy and Immune Checkpoint Blockade. Cancer Cell 2017;32:253-67.e5.

13. Kaufman HL, Kohlhapp FJ, Zloza A. Oncolytic viruses: a new class of immunotherapy drugs. Nat Rev Drug Discov 2015;14:642-62.

(English Language Editor: L. Huleatt) 